\title{
Quantitative Assessment Using the Compartment Model for Detecting Regional Coronary Artery Disease by Dynamic Myocardial Perfusion Single-Photon Emission Computed Tomography
}

\author{
Naoto Kawaguchi, MD, PhD; Masao Miyagawa, MD, PhD; Tomohisa Okada, MD; \\ Kyohei Onishi; Hayato Ishimura; Kota Tsuruoka, MD; Yuki Tanabe, MD, PhD; \\ Masashi Nakamura, MD, PhD; Tomoyuki Kido, MD, PhD; Teruhito Mochizuki, MD, PhD; \\ Toru Miyoshi, MD, PhD; Osamu Yamaguchi, MD, PhD; Teruhito Kido, MD, PhD
}

\begin{abstract}
Background: This study aimed to quantitatively evaluate myocardial perfusion single-photon emission computed tomography (SPECT) using an original analysis tool in the compartment model for detecting regional significant coronary artery disease (CAD).

Methods and Results: This study analyzed 41 patients (median age, 76 years) with suspected or known CAD who underwent both

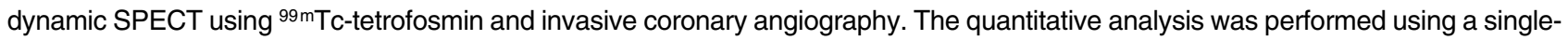
tissue compartment model to evaluate the diagnostic performance of the myocardial flow reserve (MFR) for regional significant CAD, excluding infarcted territories. In the regional analysis, 114 vessels were assessed, of which 31 were diagnosed as significant coronary lesions $(\geq 70 \%$ stenosis and/or fraction flow reserve $\leq 0.8)$. The MFR of regional significant CAD was significantly lower than that of non-significant CAD (1.11 [0.97-1.31] vs. 1.74 [1.30-2.27]; $\mathrm{P}<0.001)$. In the receiver operating characteristic curve analysis, the MFR displayed an area under the curve (AUC) of 0.81 . While analyzing each coronary artery territory, the diagnostic performance of the MFR value in the left anterior descending (LAD) artery territory was found to be significantly higher than that found in qualitative assessment (AUC: 0.84 vs. 0.61).
\end{abstract}

Conclusions: A quantitative analysis of dynamic SPECT data facilitated detecting regional CAD. For the LAD artery, the MFR displayed a higher diagnostic performance than the qualitative assessment of conventional myocardial perfusion SPECT.

Key Words: Coronary artery disease; Flow reserve; Myocardial perfusion imaging; Single photon emission computed tomography

$\mathbf{S}$ ingle-photon emission computed tomography (SPECT) myocardial perfusion imaging (MPI) with technetium-99 m-labeled $\left({ }^{99} \mathrm{~m} \mathrm{Tc}\right)$ perfusion agents is widely used in clinical practice for the assessment of coronary artery disease (CAD); however, SPECT-MPI is disadvantageous in the assessment of patients with multi-vessel disease because of balanced ischemia, ${ }^{1}$ thus necessitating a quantitative assessment of myocardial perfusion. Positron emission tomography (PET) is the gold standard for the quantitative assessment of myocardial blood flow (MBF) and myocardial flow reserve (MFR), used to detect significant CAD and predict long-term prognosis.,3

Recently, the advent of ultrafast cardiac SPECT cameras with cadmium zinc telluride (CZT) semiconductor detectors has enabled the acquisition of dynamic data with high temporal and spatial resolutions; therefore, it has facilitated the quantitative evaluation of myocardial perfusion using SPECT instead of PET. 4-12

Several researchers have compared SPECT-based quantitative myocardial perfusion with PET-based methods; however, the quantitative analysis methods used were inconsistent. Myocardial perfusion PET is often quantitatively analyzed using a compartment model; however, there are few reports on SPECT.4,5 We developed an original quantitative analysis tool using a compartment model and performed an assessment using SPECT-MPI with dynamic acquisition. Despite reports about the usefulness of global quantitative myocardial perfusion SPECT for

Received November 21, 2021; revised manuscript received January 27, 2022; accepted January 30, 2022; J-STAGE Advance Publication released online February 23, 2022 Time for primary review: 11 days

Department of Radiology (N.K., M.M., T.O., K.O., H.I., K.T., Y.T., M.N., To. Kido, T. Mochizuki, Te. Kido), Department of Cardiology (T. Miyoshi, O.Y.), Ehime University Graduate School of Medicine, Toon, Japan; Department of Radiology, I.M. Sechenov First Moscow State Medical University, Moscow (T. Mochizuki), Russia

Mailing address: Naoto Kawaguchi, MD, PhD, Department of Radiology, Ehime University Graduate School of Medicine, Shitsukawa, Toon 791-0295, Japan. E-mail: n.kawa1113@gmail.com

All rights are reserved to the Japanese Circulation Society. For permissions, please e-mail: cj@j-circ.or.jp

ISSN-1346-9843 


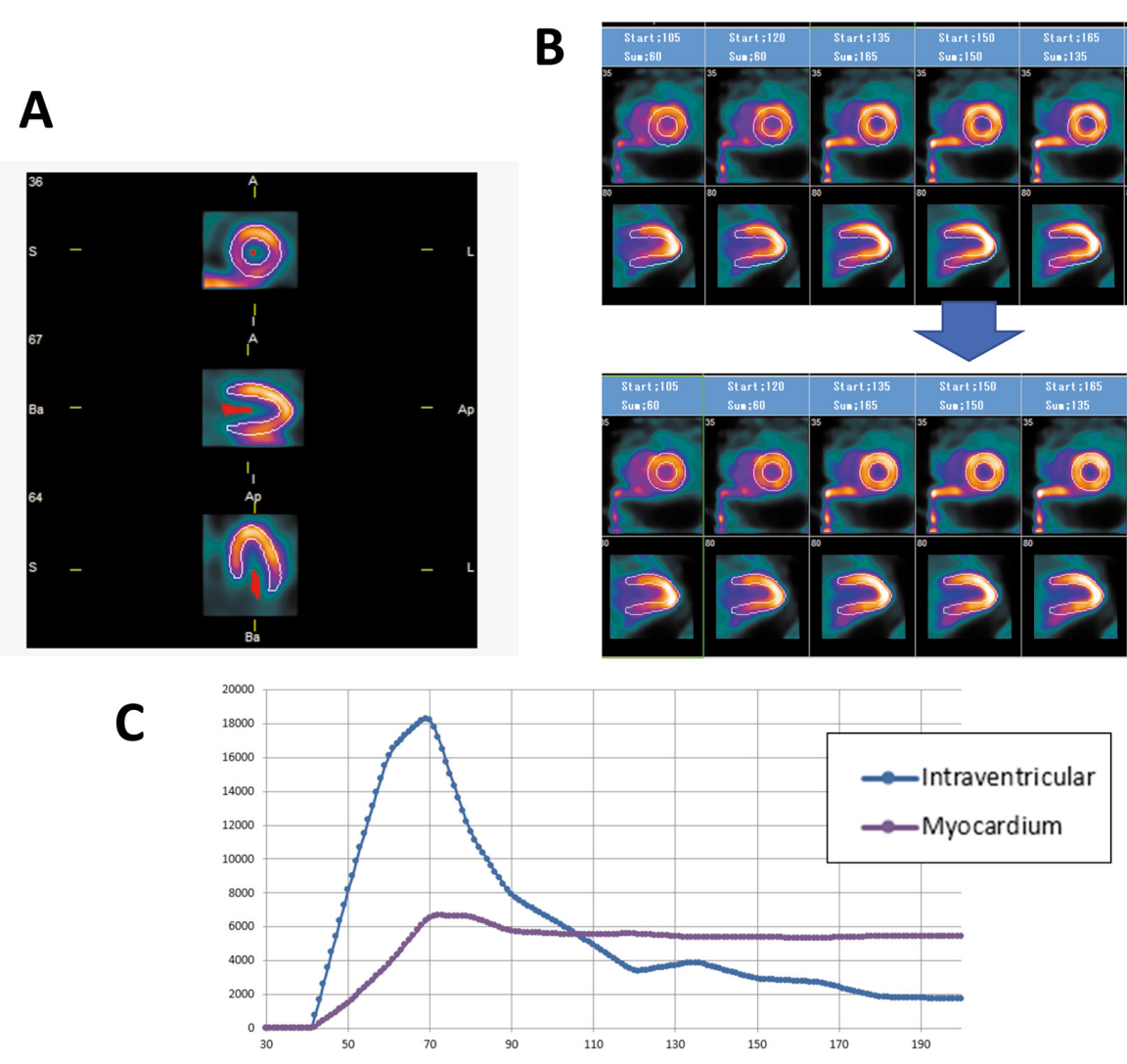

Figure 1. Quantitative analysis method using dynamic SPECT. (A) The VOI of the arterial input function is manually set at the position of the left intraventricular cavity near the mitral valve to prevent an overlap with the myocardium. (B) The position of the myocardial VOI is manually corrected for each frame for motion correction (Upper section: pre-motion correction, lower section: post-motion correction). (C) TACs are created from the obtained intraventricular (blue line) and myocardium (purple line) data, and $\mathrm{K} 1$ values are calculated using the single-tissue-compartmental model. SPECT, single photon emission computed tomography; TACs, time activity curves; VOI, volume of interest.

detecting multi-vessel disease, we did not evaluate regional CAD. , $^{7}$

This study aimed to evaluate quantitative myocardial perfusion SPECT with an original analysis tool using the compartment model for detecting regional significant CAD.

\section{Methods}

\section{Study Patients}

From January 2019 to January 2020, we performed myocardial perfusion SPECT with dynamic acquisition in 175 consecutive patients with suspected or known CAD, who were referred to the Ehime University Hospital. Of these patients, 70 underwent invasive coronary angiography (ICA) based on the decision of the attending physician within 6 months of dynamic SPECT. None of the patients experienced cardiac events between undergoing dynamic SPECT and ICA. The exclusion criteria were as follows: (1) unstable angina; (2) a history of myocardial infarction; (3) previous coronary artery bypass grafting or percutaneous coronary intervention within 90 days before SPECT; (4) atrioventricular block greater than the first degree; (5) symptomatic asthma; (6) non-ischemic cardiomyopathy; and (7) unacceptable patient body motion during dynamic data collection.

Of the 70 patients, 29 patients were withdrawn from the study based on the exclusion criteria (myocardial infarction, $n=9$; previous coronary artery bypass grafting, $n=7$; recent percutaneous coronary intervention, $n=6$; unacceptable patient body motion during dynamic data collection, $\mathrm{n}=7$ ). Eventually, we included 41 patients and subjected them to a quantitative analysis (median age, 76 years; 30 men).

The institutional ethics committee approved this study protocol in accordance with the ethical guidelines of the Declaration of Helsinki (Ehime University Hospital, No. 1810015), and all patients provided informed consent.

\section{Dynamic Myocardial Perfusion SPECT Protocol}

All patients were imaged in the supine position using a CZT camera (DNM 530c; GE Healthcare, Haifa, Israel). We performed dynamic myocardial perfusion SPECT with a stress-first 1-day protocol using $99 \mathrm{~m}$ Tc-tetrofosmin. Our dynamic protocol is shown in Supplementary Figure. First, we injected a test dose of a tracer $(0.3 \mathrm{MBq} / \mathrm{kg}$ body weight $)$ for positioning the patients for scanning. For pharmacological stress, adenosine triphosphate disodium (ATP) was infused at a rate of $180 \mu \mathrm{g} \cdot \mathrm{kg}^{-1} \cdot \mathrm{min}^{-1}$ over $5 \mathrm{~min} .{ }^{13}$ Fol- 
lowing $3 \mathrm{~min}$ of ATP infusion, we injected a tracer diluted in saline to $6-8 \mathrm{~mL}$ at a dose of $3 \mathrm{MBq} / \mathrm{kg}$. It was flushed by $23 \mathrm{~mL}$ of saline using an automatic injector (Terufusion syringe pump; Terumo, Tokyo, Japan) at a constant rate of $0.23 \mathrm{~mL} / \mathrm{s}$ for $100 \mathrm{~s}$. We initiated dynamic data acquisition with a bolus of tracer infusion and continuously recorded list-mode data. Standard electrocardiogramgated stress imaging for $5 \mathrm{~min}$ was commenced $60 \mathrm{~min}$ after tracer injection for routine clinical interpretation. More than $3 \mathrm{~h}$ later, we acquired a 300 s pre-scan to obtain the baseline data for subtracting the counts from subsequent resting data. Eventually, we began dynamic data acquisition with a bolus of $9 \mathrm{MBq} / \mathrm{kg}$. Gated rest imaging was performed similar to the stress scan. Images were reconstructed without attenuation or scatter correction.

\section{Quantification From Dynamic SPECT Data}

We performed dynamic SPECT imaging at rest and during pharmacologic stress under a 9-min acquisition protocol in the list mode, followed by the reconstruction of dynamic frames $(9 \times 10 \mathrm{~s}, 6 \times 15 \mathrm{~s}$, and $3 \times 120 \mathrm{~s})$. Subsequently, the global myocardial volume of interest (VOI) was automatically placed to obtain the time activity curves (TACs) from the dynamic image using the last $4 \mathrm{~min}$ of the frame. We manually extracted cases in which the VOI could not be extracted automatically. The VOI of the arterial input function was manually set at the position of the left intraventricular cavity near the mitral valve to prevent overlap with the myocardium in 3 directions (Figure 1A). Two operators (N.K. and T.O.) manually corrected the position of the myocardial VOI for each frame for motion correction (Figure 1B). The global myocardial VOI was divided into 3 coronary territories using the standard 17 -segment polar map. ${ }^{14}$ TACs were created from the dynamic data of intraventricular and myocardium, which were interpolated from the multi-frame to a 1-s frame. Moreover, K1 values were calculated using a single-tissue-compartment model for the global left ventricle myocardium and each coronary territory of the left anterior descending (LAD) artery, left circumflex (LCX) artery, and right coronary artery (RCA) using the standard coronary anatomy from the 17-segment model (Figure 1C). ${ }^{15} \mathrm{We}$ converted the K1 values to MBF using the following Renkin-Crone equation by proposed by Leppo: ${ }^{16}$

$$
\mathrm{K} 1=\operatorname{MBF}^{*}\left(1-\alpha * \mathrm{e}^{-\beta / \mathrm{MBF}}\right)
$$

The parameters were set at $\alpha=0.874$ and $\beta=0.443$. ${ }^{9}$

The mean MFR was calculated as the ratio of stress MBF (stress scan) to rest MBF (rest scan) in global myocardium and each coronary territory.

\section{Image Interpretation From Static SPECT Data}

Two experienced nuclear medicine readers (N.K. and M.M.) evaluated the static SPECT images. Each coronary territory was scored on a semi-quantitative 5-point scoring system (from $0=$ normal uptake to $4=$ absent radiotracer distribution). ${ }^{14}$ They quantified the sum of the stress scores of all segments (SSS) and the sum of the rest scores of all segments (SRS). The summed difference score (SDS) was calculated as the difference between the SSS and SRS. The coronary territory with a SDS $\geq 1$ was defined as the ischemic territory on the regional analysis. ${ }^{17}$ The territory that displayed a fixed defect was defined as the old myocardial infarcted territory. Each reader evaluated the diagnosis independently. The final diagnosis was decided by consen-

\begin{tabular}{|c|c|}
\hline \multicolumn{2}{|l|}{ Characteristics } \\
\hline Sex, male, $n(\%)$ & $31(76)$ \\
\hline Age (years) & $76(69-79)$ \\
\hline Body weight (kg) & $66(57-70)$ \\
\hline Body mass index $\left(\mathrm{kg} / \mathrm{m}^{2}\right)$ & $24.2(22.1-26.5)$ \\
\hline \multicolumn{2}{|l|}{ Risk factor, n (\%) } \\
\hline Hypertension & $29(71)$ \\
\hline Diabetes mellitus & $18(44)$ \\
\hline Hyperlipidemia & $21(51)$ \\
\hline Smoking & $16(39)$ \\
\hline Family history of CAD & $11(27)$ \\
\hline Chronic kidney disease & $4(10)$ \\
\hline Coronary intervention & $9(22)$ \\
\hline \multicolumn{2}{|l|}{ Symptom, n (\%) } \\
\hline Typical chest pain & $9(22)$ \\
\hline Atypical chest pain & $9(22)$ \\
\hline Chest discomfort & $6(15)$ \\
\hline Asymptomatic & $17(41)$ \\
\hline \multicolumn{2}{|l|}{ Medication, n (\%) } \\
\hline Statin & $30(73)$ \\
\hline$\beta$-blocker & $14(34)$ \\
\hline Calcium-channel blocker & $22(54)$ \\
\hline ACEI/ARB & $16(39)$ \\
\hline Aspirin & $21(51)$ \\
\hline \multicolumn{2}{|l|}{ Echocardiography } \\
\hline LVEF (\%) & $65(59-69)$ \\
\hline LVEDV (mL) & $77(56-93)$ \\
\hline LVESV (mL) & $25(20-35)$ \\
\hline \multicolumn{2}{|l|}{ SPECT and ICA examinations } \\
\hline SPECT first & $29(71)$ \\
\hline ICA first & $12(29)$ \\
\hline Interval (days) & $23(9-56)$ \\
\hline Previous cardiac CT ( $<6$ months) & $12(29)$ \\
\hline Previous PCl & $9(22)$ \\
\hline
\end{tabular}

Data are presented as medians and interquartile ranges or $\mathrm{n}(\%)$, or $\mathrm{n}(\%)$. ACEI, angiotensin-converting enzyme inhibitor; ARB, angiotensin-receptor blocker; CAD, coronary artery disease; CT, computed tomography; ICA, invasive coronary angiography; LVEDV, left ventricular end-diastolic volume; LVEF, left ventricular ejection fraction; LVESV, left ventricular end-systolic volume; $\mathrm{PCl}$, percutaneous coronary intervention; SPECT, single photon emission computed tomography.

sus in case of a disagreement between them.

\section{Invasive Coronary Angiography Analysis}

Diagnostic ICA and fractional flow reserve (FFR) measurements were recorded according to standard clinical protocols. We performed a quantitative coronary angiography (QCA) analysis of the percent diameter stenosis using QCA analysis software (Nahri AQUA; Nexis, Fukuoka, Japan). Invasive FFR measurements were performed by experienced interventional cardiologists for clinical indications following the visual assessment. The FFR measurement was performed using a catheter $>5 \mathrm{Fr}$. Intravenous ATP or nicorandil was injected into the coronary arteries to obtain maximum hyperemia.

Significant CAD was defined as coronary artery stenosis $\geq 70 \%$ on the ICA with QCA and/or FFR values $\leq 0.8$. On 


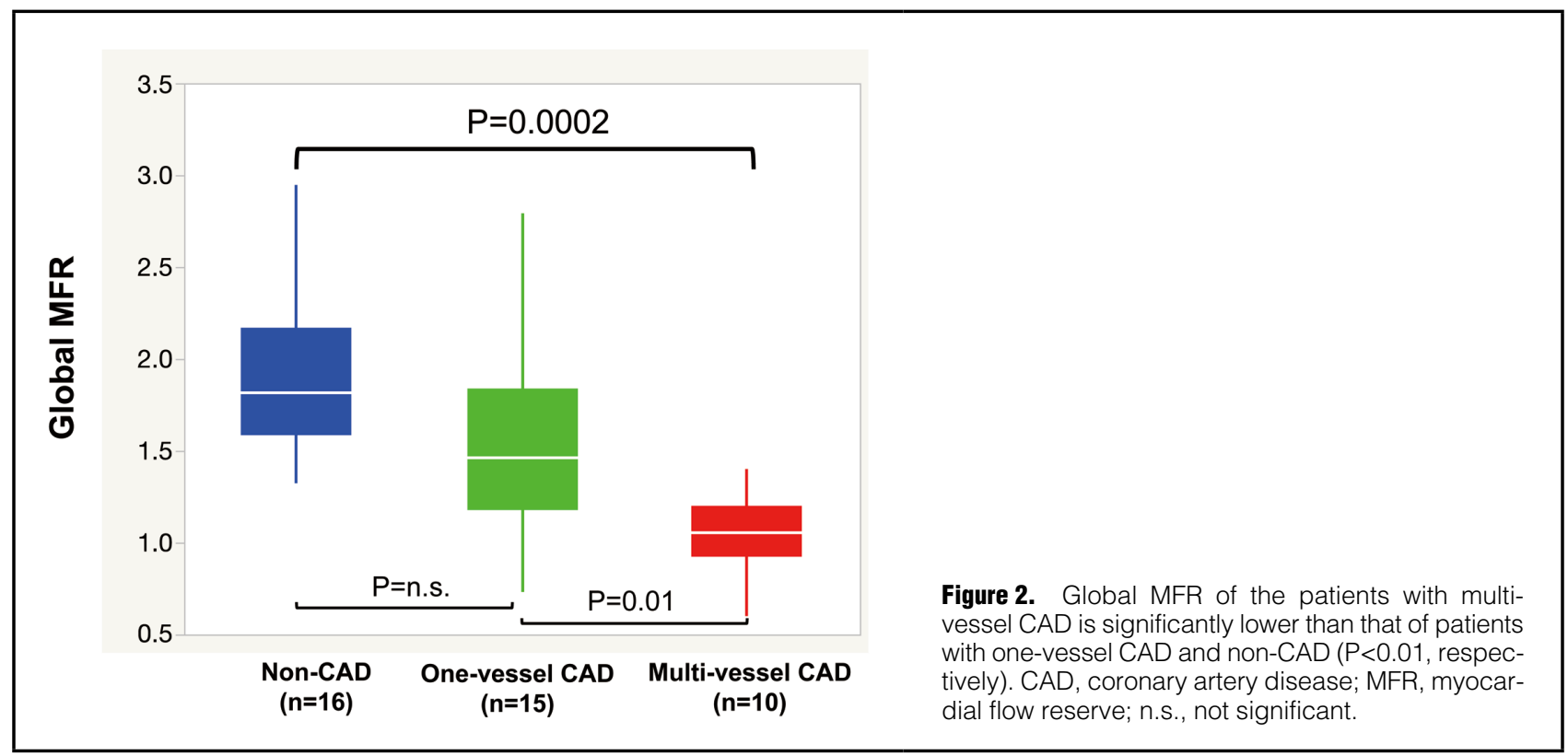

\begin{tabular}{|lccc|}
\hline \multicolumn{1}{l}{ Table 2. Regional Quantitative Values Compared Between Significant and Non-Significant Lesions } \\
& $\begin{array}{c}\text { Significant lesion } \\
(\mathbf{n = 3 1 )}\end{array}$ & $\begin{array}{c}\text { Non-significant lesion } \\
(\mathbf{n = 8 3})\end{array}$ & P value \\
Rest $\mathrm{MBF}\left(\mathrm{mL} \cdot \mathrm{min}^{-1} \cdot \mathrm{g}^{-1}\right)$ & $1.05(0.82-1.36)$ & $0.94(0.71-1.22)$ & 0.22 \\
Stress $\mathrm{MBF}\left(\mathrm{mL} \cdot \mathrm{min}^{-1} \cdot \mathrm{g}^{-1}\right)$ & $1.13(0.87-1.51)$ & $1.63(1.17-2.41)$ & 0.0005 \\
MFR & $1.11(0.97-1.31)$ & $1.74(1.30-2.27)$ & $<0.0001$ \\
\hline
\end{tabular}

Data are presented as medians and interquartile ranges. MBF, myocardial blood flow; MFR, myocardial flow reserve.

performing FFR measurements during ICA, stenosis with FFR values $>0.8$ was classified as non-significant. In contrast, in the absence of FFR, coronary stenosis with QCA values $<70 \%$ was classified as non-significant. ${ }^{18}$

\section{Statistical Analyses}

Continuous variables are expressed as median and interquartile range, or $\mathrm{n}(\%)$. We performed linear regression and Bland-Altman analysis with the calculation of the repeatability coefficient $(\mathrm{RPC}=1.96 \times$ standard deviation of differences) to evaluate the intra- and inter-observer variabilities in regional MBF and MFR. The global quantitative parameters of myocardial perfusion were compared using the Steel-Dwass test. In contrast, the regional quantitative parameters of myocardial perfusion were compared using the Wilcoxon rank-sum test. The optimal cut-off values of stress MBF and MFR for the detection of significant lesions were obtained through receiver operating characteristic (ROC) analyses. Furthermore, we compared the area under the curves (AUCs) using a previously described method with Bonferroni's correction. ${ }^{\mathbf{1 9}, 20}$ The diagnostic performance was assessed using the sensitivity, specificity, positive predictive value (PPV), negative predictive value (NPV), and accuracy on a regional analysis. Statistical analyses were performed using JMP12 statistical software (SAS Institute, Cary, NC, USA). Statistical significance was set at $\mathrm{P}<0.05$. When comparing the 3 groups, $\mathrm{P}<0.0167$ was considered statistically significant using Bonferroni's correction.

\section{Results}

Table 1 summarizes the clinical characteristics of the patients. Based on data from the International Commission on Radiological Protection publications 128, ${ }^{21}$ the radiation effective dose of the entire protocol, including the test dose, was estimated to be $<9 \mathrm{mSv}$. On a regional analysis, we excluded 9 territories with fixed defects and analyzed 114 territories. FFR was calculated for 18 vessels (14 patients) and the values were $\leq 0.80$ and $>0.80$ in 10 and 8 vessels, respectively. Of the remaining 96 vessels, coronary stenosis based on QCA was $\geq 70 \%$ and $<70 \%$ in 21 and 75 vessels $(50-69 \%, 14$ vessels; $30-49 \%, 8$ vessels; and $0-29 \%, 53$ vessels), respectively. Thus, 31 vessels $(27 \%)$ had regional significant coronary lesions and 83 vessels $(73 \%)$ had nonsignificant lesions.

Of the 41 patients, 25 had significant CAD, with 1-vessel disease (1-vessel CAD) in 15 patients and 2- or 3-vessel disease (multi-vessel CAD) in 10 patients. Sixteen patients had no significant disease in any vessel (non-CAD).

\section{Quantitative Analysis of Global Myocardial Perfusion}

The global stress MBF of patients with non-CAD, 1-vessel CAD, and multi-vessel CAD were $2.11 \mathrm{~mL} \cdot \mathrm{min}^{-1} \cdot \mathrm{g}^{-1}(1.70$ 2.64), $1.51 \mathrm{~mL} \cdot \mathrm{min}^{-1} \cdot \mathrm{g}^{-1}(1.16-1.90)$, and $1.09 \mathrm{~mL} \cdot \mathrm{min}^{-1} \cdot \mathrm{g}^{-1}$ (0.99-1.35), respectively. The global rest MBF of patients with non-CAD, 1-vessel CAD, and multi-vessel CAD were $1.22 \mathrm{~mL} \cdot \mathrm{min}^{-1} \cdot \mathrm{g}^{-1}(0.81-1.46), 0.89 \mathrm{~mL} \cdot \mathrm{min}^{-1} \cdot \mathrm{g}^{-1}(0.66-$ $1.37)$, and $1.08 \mathrm{~mL} \cdot \mathrm{min}^{-1} \cdot \mathrm{g}^{-1}(0.91-1.50)$, respectively. 
Global MFR of patients with non-CAD, 1-vessel CAD, and multi-vessel CAD were 1.82 (1.58-2.17), 1.46 (1.181.84), and 1.05 (0.92-1.20), respectively. Global MFR of patients with multi-vessel CAD was significantly lower than that of patients with 1-vessel CAD and non-CAD $(\mathrm{P}=0.01$ and 0.0002 , respectively; Figure 2).

\section{Regional Quantitative Analysis of Significant and Non-Significant Lesions}

During a regional analysis, 31 of the 114 territories were diagnosed as significant lesions and 83 were diagnosed as non-significant lesions; then, the regional quantitative values of each territory were compared (Table 2). MFR and stress $\mathrm{MBF}$ were significantly lower in territory vessels with significant coronary lesion than those without significant lesion (1.11 [0.97-1.31] vs. 1.74 [1.30-2.27] and 1.13 [0.87$1.51] \mathrm{mL} \cdot \mathrm{min}^{-1} \cdot \mathrm{g}^{-1}$ vs. $1.63[1.17-2.41] \mathrm{mL} \cdot \mathrm{min}^{-1} \cdot \mathrm{g}^{-1}$ [all, $\mathrm{P}<0.001])$. MBF at rest was not significantly different between patients with and without significant CAD (1.05 [0.82$1.36]$ vs. $0.94[0.71-1.22][\mathrm{P}=0.22])$.

Intra- and inter-observer variabilities demonstrated high correlation coefficients with regression slopes of 0.98 and 0.97 for regional $\mathrm{MBF}$ and 0.96 and 0.95 for regional MFR. Although the RPC for regional MBF was $0.20 \mathrm{~mL} \cdot \mathrm{min}^{-1} \cdot \mathrm{g}^{-1}$ and $0.26 \mathrm{~mL} \cdot \mathrm{min}^{-1} \cdot \mathrm{g}^{-1}$, that for regional MFR was 0.30 and 0.41 .

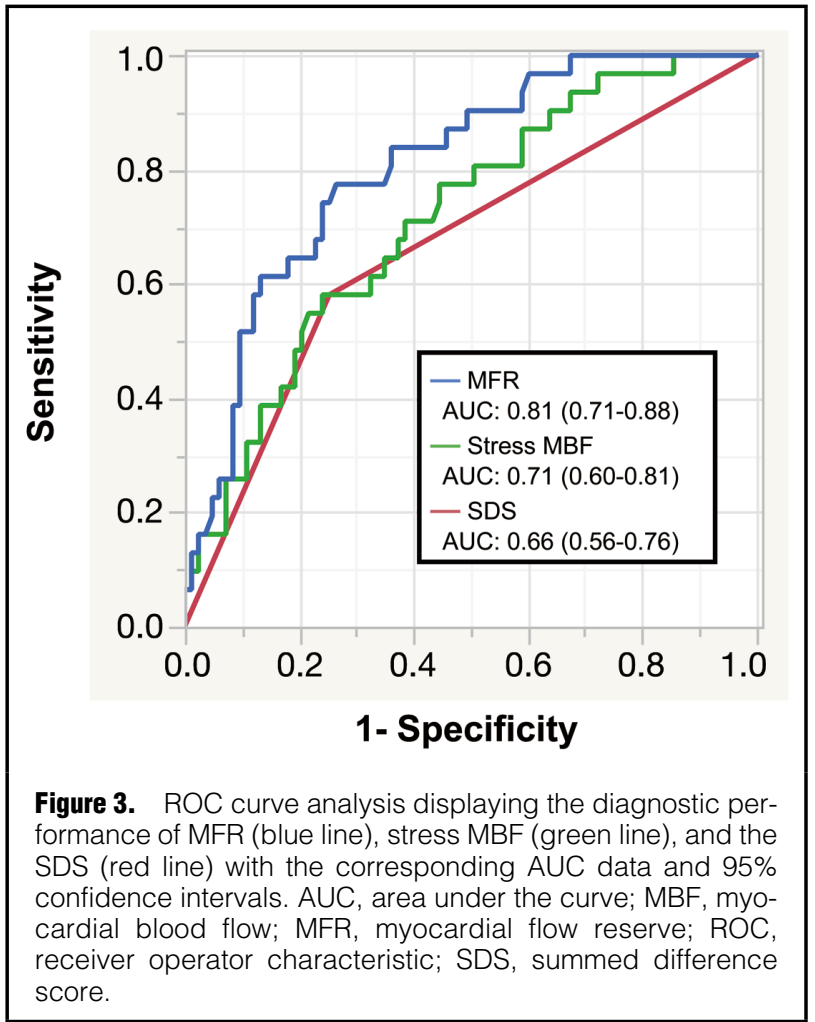

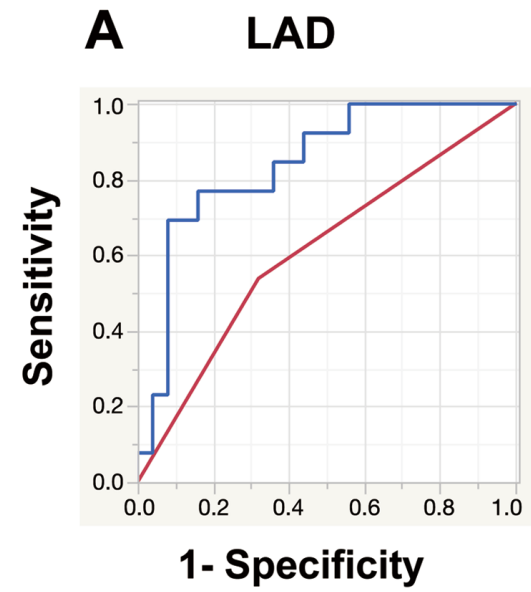
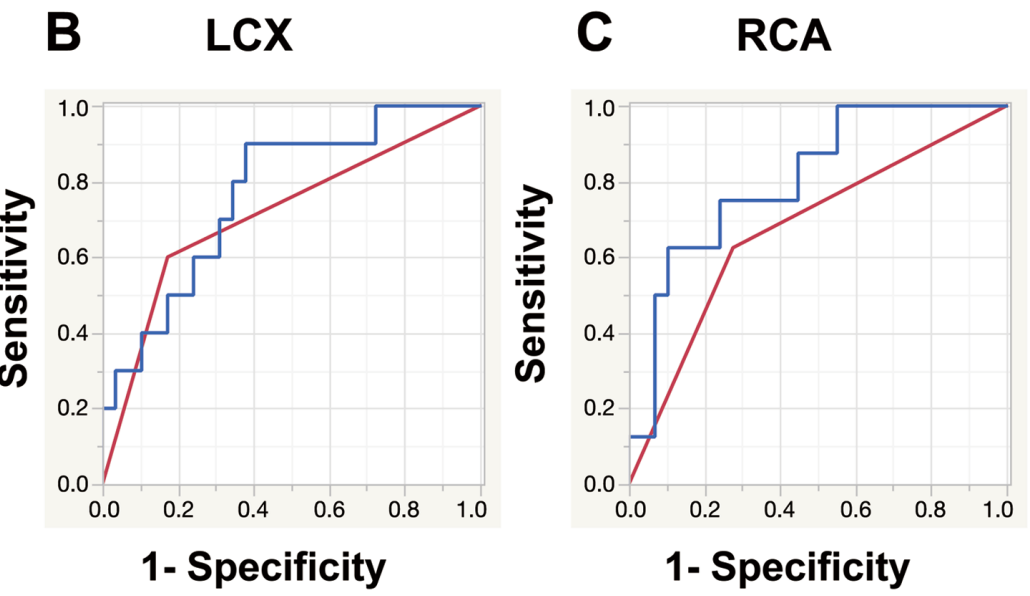

Figure 4. The ROC curve analysis for each coronary territory displaying the diagnostic performance of MFR (blue line) and the SDS (red line). (A) LAD artery. (B) LCX artery. (C) RCA. LAD, left anterior descending; LCX, left circumflex; MFR, myocardial flow reserve; RCA, right coronary artery; ROC, receiver operator characteristic; SDS, summed difference score.

\begin{tabular}{|lcccccc|}
\hline \multicolumn{2}{|c}{ Table 3. Comparison of the Diagnostic Performance Between MFR and SDS (Each Coronary Artery Territory) } \\
& $\begin{array}{c}\text { Significant } \\
\text { CAD }\end{array}$ & $\begin{array}{c}\text { Non-significant } \\
\text { CAD }\end{array}$ & P value & AUC (MFR) & AUC (SDS) & $\begin{array}{c}\text { P value } \\
\text { (MFR vs. SDS) }\end{array}$ \\
LAD & $1.11(0.93-1.36)$ & $1.80(1.33-2.28)$ & 0.0007 & $0.84(0.66-0.93)$ & $0.61(0.43-0.76)$ & 0.047 \\
LCX & $1.18(0.53-1.33)$ & $1.59(1.21-2.16)$ & 0.013 & $0.72(0.51-0.85)$ & $0.71(0.51-0.85)$ & 0.69 \\
RCA & $1.12(1.05-1.53)$ & $1.74(1.32-2.39)$ & 0.0093 & $0.81(0.59-0.92)$ & $0.67(0.49-0.83)$ & 0.24 \\
\hline
\end{tabular}

Continuous variables are presented as medians and interquartile ranges; the diagnostic performance expressed with corresponding area under the curve (AUC) data and 95\% confidence intervals. CAD, coronary artery disease; LAD, left anterior descending artery; LCX, left circumflex artery; MFR, myocardial flow reserve; RCA, right coronary artery; SDS, summed difference score. 


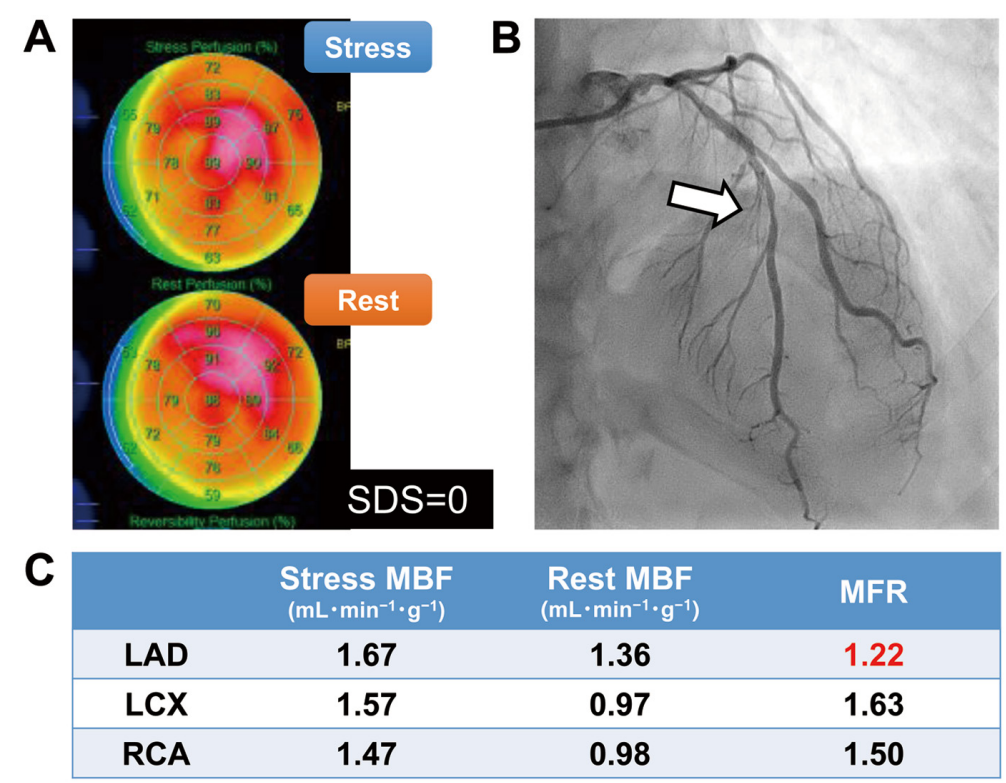

Figure 5. A representative case. A woman aged in her $60 \mathrm{~s}$ with suspected coronary artery disease had undergone dynamic SPECT and ICA. (A) Myocardial perfusion images. (B) Left coronary angiography. (C) Quantitative myocardial perfusion SPECT parameters. The parameters of significant coronary artery disease using our optimal cut-off value are displayed in red. Quantitative coronary angiography reveals intermediate stenosis (48\% stenosis) and hemodynamic significance (FFR: 0.78) at the LAD artery (white arrow). No significant stenosis is observed in the LCX artery and RCA. The SDS by myocardial perfusion images is 0 , indicating a false-negative value for the LAD artery. MFR is able to diagnose 1-vessel disease of functionally significant stenosis in the LAD artery. FFR, fractional flow reserve; ICA, invasive coronary angiography; LAD, left anterior descending; LCX, left circumflex; MBF, myocardial blood flow; MFR, myocardial flow reserve; RCA, right coronary artery; SDS, summed difference score; SPECT, single photon emission computed tomography.

\section{Diagnostic Performance of Quantitative and Qualitative Analysis}

The ROC analysis revealed an AUC of $0.81,0.71$, and 0.66 for MFR, stress MBF, and the SDS, respectively $(\mathrm{P}=0.059$ for MFR vs. stress MBF, $\mathrm{P}=0.038$ for MFR vs. SDS, and $\mathrm{P}=0.47$ for stress MBF vs. SDS; Figure 3). The optimal cut-off values for MFR and stress MBF were 1.31 and $1.36 \mathrm{~mL} \cdot \mathrm{min}^{-1} \cdot \mathrm{g}^{-1}$, respectively. MFR displayed a higher diagnostic performance in detecting significant CAD than stress MBF and the SDS. Nonetheless, the difference was not statistically significant among the 3 groups.

The sensitivity, specificity, PPV, NPV, and accuracy of the SDS, stress MBF, and MFR to detect regional significant CAD were $58 \%, 75 \%, 46 \%, 83 \%$, and $70 \%$ for the SDS, $71 \%, 61 \%, 41 \%, 85 \%$, and $64 \%$ for stress MBF, and $77 \%, 75 \%, 53 \%, 90 \%$, and $75 \%$ for MFR, respectively.

\section{Analyzing Each Coronary Artery Territory}

Overall, $13(34.2 \%), 10(25.6 \%)$, and $9(21.6 \%)$ vessels demonstrated significant CAD in the LAD artery, LCX artery, and RCA territories, respectively. Figure $\mathbf{4}$ and Table 3 depict the MFR values and diagnostic performance for each coronary artery territory in comparison between MFR and the SDS. The MFR values of the LAD artery, LCX artery, and RCA were significantly lower in the territory comprising vessels with significant lesions than in those without significant CAD (1.11 [0.93-1.36] vs. 1.80 [1.33-2.28], 1.18 [0.53-1.33] vs. 1.59 [1.21-2.16], and 1.12 [1.05-1.53] vs. 1.74 [1.32-2.39] [ $\mathrm{P}=0.0007,0.013$, and 0.0093 , respectively]). Between MFR and the SDS, the diagnostic performance of MFR to detect significant CAD was significantly higher only in the LAD artery territory (AUC: 0.84 vs. $0.61, \mathrm{P}=0.047$ ).

Figure 5 depicts a representative case.

\section{Discussion}

The MFR value determined by the quantitative analysis using the compartment model obtained by dynamic CZTSPECT was useful for diagnosing not only multi-vessel CAD but also regional significant coronary lesions. First, MFR and stress MBF were significantly low in areas with significant coronary lesions determined by QCA, including FFR measurements. Subsequently, we performed an ROC analysis on the diagnostic performance to detect each coronary lesion. The AUC of MFR was the highest among MFR, stress MBF, and the semi-quantitative SDS on qualitative SPECT. Eventually, the diagnostic performance of the MFR value in the LAD artery region was significantly higher than that of the qualitative assessment on CZT-SPECT; however, the LCX artery and RCA regions were comparable.

Myocardial perfusion SPECT involves quantification by PET owing to advances in equipment and improvements in quantitative analysis methods. The advent of CZTSPECT has facilitated obtaining high-quality 3D images with high sensitivity and resolution, thus enabling dynamic acquisition. Researchers have reported on the usefulness of a quantitative assessment of myocardial perfusion by dynamic CZT-SPECT., , , , , 10,12 There are different methods of quan- 
titative myocardial SPECT, including the type of CZT gamma cameras, tracers, dynamic acquisition protocols, and quantification analysis. Shiraishi et $\mathbf{a l}^{\mathbf{6}}$ and Miyagawa et $\mathrm{al}^{4}$ reported on the use of thallium-201 and technetium- $-99 \mathrm{~m}$ perfusion agents, respectively, for determining the usefulness of MFR in detecting multi-vessel disease using identical CZT gamma cameras and quantitative analysis with a 1-tissue compartment model, similar to that used in the current study. However, the diagnostic performance of the aforementioned method for significant CAD in each coronary region has not yet been evaluated.

One of the major disadvantages of myocardial perfusion SPECT is that it occasionally underestimates ischemia in multi-vessel disease because of balanced ischemia.1,22 This necessitates the quantitative evaluation of myocardial perfusion for SPECT and PET. First, we confirmed that global MFR with multi-vessel CAD was significantly lower than that with non-CAD and that it was useful in detecting multi-vessel disease, similar to the findings of previous reports., ${ }^{4,6}$ Subsequent regional analysis demonstrated that MFR displayed a higher diagnostic performance for significant CAD than stress $\mathrm{MBF}$ or the semi-quantitative SDS. The diagnostic performance of MFR values in the LAD artery territory was significantly higher than that of the SDS (AUC: 0.84 vs. $0.61, \mathrm{P}<0.05$ ). Chikamori et al conducted a multicenter study of 1,000 patients for the qualitative diagnostic performance of CZT-SPECT for ischemic heart disease. ${ }^{17}$ They expressed the concern that the sensitivity of CZT SPECT with Tc-99 $\mathrm{m}$ perfusion tracers for significant coronary stenoses in the LAD artery territory was lower than that with thallium-201. This was probably a consequence of the lower extraction fraction of Tc-99m perfusion tracers. Our findings suggest that an additional evaluation by MFR may add incremental values for the diagnostic performance for $\mathrm{CAD}$ in the $\mathrm{LAD}$ region.

There are several reports of quantitative analysis with MFR using CZT-SPECT to assess significant CAD. ${ }^{\mathbf{7 , 8 , 1 0 , 1 2}}$ Zavadovsky et al reported that MFR may be clinically more powerful than MBF because of several factors, including potential inaccurate extraction fraction correction, the lack of attenuation correction, differences in spatial resolution, and higher image noise. ${ }^{23}$ Considering the attenuation of the patient was similar between stress and rest, the MFR obtained by dividing the tracer uptake during the stress scan by that during the resting scan may cancel the effects of the attenuation. Moreover, a recent study reported that the MFR value of each coronary artery obtained through CZT-SPECT correlates with invasive FFR. ${ }^{10}$

In the present study, we used an original analysis tool to obtain TACs by dynamic acquisition and calculated the quantitative values using the 1-tissue compartment model to enable an accurate quantitative assessment. In the quantitative analysis by dynamic CZT-SPECT, the 1-tissue compartment model and the net retention model are the most commonly used methods. The compartment model is commonly used for the quantitative analysis in PET, and the estimate error is less than that of the retention model because of small correction factors. ${ }^{23,24}$ Thus, we used the compartment model; however, an accurate TAC is required to obtain correct $\mathrm{K} 1$ values. Therefore, we obtained TACs by manually optimizing the myocardial VOI position.

During dynamic acquisition, the influence of motion is large in the initial stage of acquisition. Moreover, motion correction by optimizing the VOI is essential for an accurate quantitative analysis. Otaki et al reported that the MBF obtained by CZT-SPECT demonstrated better correlation with that obtained by ${ }^{15} \mathrm{O}$-water PET measurements for appropriately performed motion correction. ${ }^{5}$ Similarly, the optimization of the VOI for motion correction was required at varying degrees in most cases in our study.

Accurate quantitative analyses are more difficult in the RCA and LCX artery regions than in the LAD region. This is because the effects of attenuation and extracardiac accumulation, including that in the liver, and patient motion are greater than those in the LAD region. Otaki et al mentioned that MBF obtained by CZT-SPECT was lower in the RCA and LCX regions than that by ${ }^{15} \mathrm{O}$-water PET; however, attenuation artifacts by CZT-SPECT may have influenced $\mathrm{MBF}$ measurements in these regions. ${ }^{5}$ Attenuation correction can be properly performed by X-ray computed tomography (CT) in the combined PET and CT device. Therefore, the quantification of MBF and MFR in the RCA and LCX artery regions can be performed accurately. It may also be performed with SPECT in future by using a combined SPECT and CT device.

Another technical consideration for the quantitative analysis with dynamic CZT-SPECT is to minimize the spillover of activity from the blood pool to the myocardium. Our dynamic SPECT protocol may have reduced the spillover to the VOI in the myocardium by an appropriate low dose per body weight and slow injection. This enabled a smooth TAC of the left ventricular myocardium with low radiation exposure to be obtained. In addition, the measured values of MBF and MFR demonstrated sufficiently good reproducibility within and between observers, thereby facilitating clinical application; however, we did not directly compare the quantitative values obtained using the method involving slow injection with a diluted tracer and one-shot injection. Therefore, the establishment of the optimal tracer injection method for quantification should be achieved in the future.

Recently, a large clinical trial has demonstrated that "invasive compared with conservative strategies do not improve the risk of ischemic cardiovascular events or death from any cause among patients with stable coronary disease and moderate or severe ischemia". ${ }^{25}$ In several cases in the aforementioned trial, the researchers used semiquantitative defect scores in conventional myocardial SPECT rather than quantitative assessment to assess eligible patients with moderate or severe ischemia. In contrast, myocardial perfusion PET is the gold standard for the quantification of MBF and MFR. Numerous studies have been conducted on the long-term prognosis of cardiac events based on these physiological indicators., ${ }^{2,3,26,27}$ Moreover, FFR is the current invasive standard for physiological stenosis severity, and has a history of being initially validated in comparison to quantitative PET. ${ }^{28}$

During the present study, we used FFR as a reference for evaluating regional ischemia, but FFR and MFR do not necessarily agree. ${ }^{2}$ In particular, a diffuse decrease in MFR may indicate not only multi-vessel coronary disease but also microvascular dysfunction caused by diabetes mellitus, hypertension, and hyperlipidemia. Our goal was not to obtain MFR using SPECT as a substitute for FFR. In clinical practice, patients who undergo SPECT are at high risk for CAD; therefore, it is important to be able to correctly diagnose significant ischemia to determine the optimal treatment. The qualitative assessment using SPECT 
provides abundant evidence for patients with CAD. Currently, there are various modalities to evaluate ischemia. However, by adding a new quantitative assessment to myocardial perfusion SPECT, the role of MPI should change from qualitatively assessing the ischemic area to comprehensively assessing the ischemic area, determining reperfusion therapy, and improving the patient prognosis. This necessitates the quantitative assessment of myocardial perfusion and quantification by SPECT, which can be used in facilities other than PET. Our findings indicated the potential of quantitative assessment for regional and global coronary lesions, and further prospective studies will enhance the clinical usefulness of ischemic diagnosis.

\section{Study Limitations}

The present study had some limitations. First, this study was a retrospective and included a small sample size selected from a single institution. Second, we calculated MBF and MFR of each coronary territory in a fixed territory based on 17 standard myocardium segments. It did not perfectly correspond to the actual territory of the coronary artery for individual cases; therefore, the quantitative values for ischemic and non-ischemic territories could potentially be mixed. ${ }^{29,30}$ Third, we did not measure FFR for all vessels. FFR was typically measured for a vessel with visually suspected intermediate stenosis at the operator's discretion. Regarding intermediate stenosis, the FAME study subanalysis using FFR showed that $35 \%$ of cases in the $50-70 \%$ stenosis category were functionally significant. ${ }^{31}$ This may underestimate the number of cases of significant coronary lesions.

Finally, the patients included in present study had a higher prevalence of CAD. Because some patients had normal findings on myocardial SPECT, they did not undergo ICA. Additionally, myocardial SPECT has been used with ICA to assess ischemia in patients with stenosis. Compared to evaluations of consecutive patients with suspected CAD, this may have led to selection bias.

\section{Conclusions}

In summary, it was feasible to detect regional CAD by using quantitative analysis of dynamic CZT SPECT data. In the LAD artery region, the MFR values demonstrated higher diagnostic performance than the qualitative assessment of conventional myocardial perfusion SPECT.

\section{Acknowledgments}

The authors are grateful to Motomu Shirai and Kazumasa Nishida of Nihon Medi-Physics Co. (Tokyo, Japan) for improving the quantitative analysis tool of dynamic myocardial perfusion SPECT.

\section{Disclosures}

The authors declare that there are no conflicts of interest.

\section{IRB Information}

The institutional ethics committee approved this study protocol, which adhered to the ethical guidelines of the Declaration of Helsinki (Ehime University Hospital, No. 1810015).

\section{Data Availability}

The deidentified participant data will not be shared.

\section{References}

1. Berman DS, Kang X, Slomka PJ, Gerlach J, de Yang L, Hayes
SW, et al. Underestimation of extent of ischemia by gated SPECT myocardial perfusion imaging in patients with left main coronary artery disease. $J$ Nucl Cardiol 2007; 14: 521-528.

2. Danad I, Uusitalo V, Kero T, Saraste A, Raijmakers PG, Lammertsma AA, et al. Quantitative assessment of myocardial perfusion in the detection of significant coronary artery disease: Cutoff values and diagnostic accuracy of quantitative $\left[{ }^{15} \mathrm{O}\right] \mathrm{H}_{2} \mathrm{O}$ PET Imaging. J Am Coll Cardiol 2014; 64: 1464-1475.

3. Herzog BA, Husmann L, Valenta I, Gaemperli O, Siegrist PT, Tay FM, et al. Long-term prognostic value of ${ }^{13} \mathrm{~N}$-ammonia myocardial perfusion positron emission tomography added value of coronary flow reserve. $J$ Am Coll Cardiol 2009; 54: 150-156.

4. Miyagawa M, Nishiyama Y, Uetani T, Ogimoto A, Ikeda S, Ishimura $\mathrm{H}$, et al. Estimation of myocardial flow reserve utilizing an ultrafast cardiac SPECT: Comparison with coronary angiography, fractional flow reserve, and the SYNTAX score. Int $J$ Cardiol 2017; 244: 347-353.

5. Otaki Y, Manabe O, Miller RJH, Manrique A, Nganoa C, Roth N, et al. Quantification of myocardial blood flow by CZT-SPECT with motion correction and comparison with ${ }^{15} \mathrm{O}$-water PET. $J$ Nucl Cardiol 2021; 28: 1477-1486.

6. Shiraishi S, Sakamoto F, Tsuda N, Yoshida M, Tomiguchi S, Utsunomiya D, et al. Prediction of left main or 3-vessel disease using myocardial perfusion reserve on dynamic thallium-201 single-photon emission computed tomography with a semiconductor gamma camera. Circ J 2015; 79: 623-631.

7. Zavadovsky KV, Mochula AV, Maltseva AN, Boshchenko AA, Baev AE, Andreev SL, et al. The diagnostic value of SPECT CZT quantitative myocardial blood flow in high-risk patients. $J$ Nucl Cardiol, doi:10.1007/s12350-020-02395-8.

8. Agostini D, Roule V, Nganoa C, Roth N, Baavour R, Parienti JJ, et al. First validation of myocardial flow reserve assessed by dynamic ${ }^{99} \mathrm{~m}$ Tc-sestamibi CZT-SPECT camera: Head to head comparison with ${ }^{15} \mathrm{O}$-water PET and fractional flow reserve in patients with suspected coronary artery disease. The WATERDAY study. Eur J Nucl Med Mol Imaging 2018; 45: 1079-1090.

9. Giubbini R, Bertoli M, Durmo R, Bonacina M, Peli A, Faggiano I, et al. Comparison between $\mathrm{N}^{13} \mathrm{NH}_{3}-\mathrm{PET}$ and ${ }^{99} \mathrm{mTc}$-TetrofosminCZT SPECT in the evaluation of absolute myocardial blood flow and flow reserve. J Nucl Cardiol 2021; 25: 1906-1908.

10. Zavadovsky KV, Mochula AV, Boshchenko AA, Vrublevsky AV, Baev AE, Krylov AL, et al. Absolute myocardial blood flows derived by dynamic CZT scan vs invasive fractional flow reserve: Correlation and accuracy. $J$ Nucl Cardiol 2021; 28: 249-259.

11. Hyafil F, Chequer R, Sorbets E, Estellat C, Ducrocq G, Rouzet $F$, et al. Head-to-head comparison of the diagnostic performances of Rubidium-PET and SPECT with CZT camera for the detection of myocardial ischemia in a population of women and overweight individuals. $J$ Nucl Cardiol 2020; 27: 755-768.

12. Li C, Xu R, Yao K, Zhang J, Chen S, Pang L, et al. Functional significance of intermediate coronary stenosis in patients with single-vessel coronary artery disease: A comparison of dynamic SPECT coronary flow reserve with intracoronary pressurederived fractional flow reserve (FFR). J Nucl Cardiol, doi:10.1007/s12350-020-02293-z.

13. Miyagawa M, Kumano S, Sekiya M, Watanabe K, Akutzu H, Imachi $\mathrm{T}$, et al. Thallium-201 myocardial tomography with intravenous infusion of adenosine triphosphate in diagnosis of coronary artery disease. J Am Coll Cardiol 1995; 26: 11961201.

14. Cerqueira MD, Weissman NJ, Dilsizian V, Jacobs AK, Kaul S, Laskey WK, et al. Standardized myocardial segmentation and nomenclature for tomographic imaging of the heart: A statement for healthcare professionals from the Cardiac Imaging Committee of the Council on Clinical Cardiology of the American Heart Association. Circulation 2002; 105: 539-542.

15. Ben-Haim S, Murthy VL, Breault C, Allie R, Sitek A, Roth N, et al. Quantification of myocardial perfusion reserve using dynamic SPECT imaging in humans: A feasibility study. $J$ Nucl Med 2013; 54: 873-879.

16. Leppo JA, Meerdink DJ. Comparison of the myocardial uptake of a technetium-labeled isonitrile analogue and thallium. Circ Res 1989; 65: 632-639.

17. Chikamori T, Goto K, Hida S, Miyagawa M, Ishimura H, Uchida $\mathrm{K}$, et al. Diagnostic performance of a semiconductor gammacamera system as studied by multicenter registry. J Cardiol 2017; 69: $449-455$.

18. Kawaguchi N, Okayama H, Kawamura G, Shigematsu T, Takahashi T, Kawada Y, et al. Clinical usefulness of coronary flow reserve ratio for the detection of significant coronary artery 
disease on ${ }^{13} \mathrm{~N}$-ammonia positron emission tomography. Circ $J$ 2018; 82: 486-493.

19. DeLong ER, DeLong DM, Clarke-Pearson DL. Comparing the areas under two or more correlated receiver operating characteristic curves: A nonparametric approach. Biometrics 1988; 44: $837-845$

20. Shaffer JP. Multiple hypothesis testing. Annu Rev Psychol 1995; 46: $561-584$.

21. Mattsson S, Johansson L, Leide Svegborn S, Liniecki J, Noßke $\mathrm{D}$, Riklund $\mathrm{KÅ}$, et al. Radiation dose to patients from radiopharmaceuticals: A compendium of current information related to frequently used substances. Ann ICRP 2015; 44: 7-321.

22. Driessen RS, Danad I, Stuijfzand WJ, Raijmakers PG, Schumacher SP, van Diemen PA, et al. Comparison of coronary computed tomography angiography, fractional flow reserve, and perfusion imaging for ischemia diagnosis. J Am Coll Cardiol 2019; 73: $161-173$.

23. Zavadovsky KV, Mochula AV, Maltseva AN, Shipulin VV, Sazonova SI, Gulya MO, et al. The current status of CZT SPECT myocardial blood flow and reserve assessment: Tips and tricks. J Nucl Cardiol, doi:10.1007/s12350-021-02620-y.

24. Lortie M, Beanlands RS, Yoshinaga K, Klein R, Dasilva JN, DeKemp RA. Quantification of myocardial blood flow with ${ }^{82} \mathrm{Rb}$ dynamic PET imaging. Eur J Nucl Med Mol Imaging 2007; 34: $1765-1774$.

25. Maron DJ, Hochman JS, Reynolds HR, Bangalore S, O'Brien $\mathrm{SM}$, Boden WE, et al. Initial invasive or conservative strategy for stable coronary disease. N Engl J Med 2020; 382: 1395-1407.

26. Gould KL, Johnson NP, Roby AE, Nguyen T, Kirkeeide R, Haynie M, et al. Regional, artery-specific thresholds of quantita- tive myocardial perfusion by PET associated with reduced myocardial infarction and death after revascularization in stable coronary artery disease. $J$ Nucl Med 2018; 60: 410-417.

27. Gould KL, Kitkungvan D, Johnson NP, Nguyen T, Kirkeeide $\mathrm{R}$, Bui L, et al. Mortality prediction by quantitative PET perfusion expressed as coronary flow capacity with and without revascularization. JACC Cardiovasc Imaging 2021; 14: 1020-1034.

28. De Bruyne B, Baudhuin T, Melin JA, Pijls NH, Sys SU, Bol A, et al. Coronary flow reserve calculated from pressure measurements in humans: Validation with positron emission tomography. Circulation 1994; 89: 1013-1022.

29. Ortiz-Perez JT, Rodriguez J, Meyers SN, Lee DC, Davidson C, $\mathrm{Wu}$ E. Correspondence between the 17-segment model and coronary arterial anatomy using contrast-enhanced cardiac magnetic resonance imaging. J Am Coll Cardiol 2008; 1: 282-293.

30. Pereztol-Valdes O, Candell-Riera J, Santana-Boado C, Angel J, Aguade-Bruix S, Castell-Conesa J, et al. Correspondence between left ventricular 17 myocardial segments and coronary arteries. Eur Heart J 2005; 26: 2637-2643.

31. Tonino PA, Fearon WF, De Bruyne B, Oldroyd KG, Leesar MA, Ver Lee PN, et al. Angiographic versus functional severity of coronary artery stenoses in the FAME study fractional flow reserve versus angiography in multivessel evaluation. $\mathrm{J} \mathrm{Am} \mathrm{Coll}$ Cardiol 2010; 55: 2816-2821.

\section{Supplementary Files}

Please find supplementary file(s); http://dx.doi.org/10.1253/circj.CJ-21-0966 